\title{
Optimization of the Method of Auxiliary Sources by the Genetic Algorithm for electromagnetic scattering problem
}

\author{
Sami Hidouri and Taoufik Aguili \\ Syscom Laboratory, National Engineering School, B.P 37 Le belvedere 1002 Tunis, Tunisia \\ *corresponding author, E-mail: hidouri_sami@yahoo.fr
}

\begin{abstract}
In this paper, we propose to apply a technique of optimization of the convergence of the method of auxiliary sources (MAS) for the 2D problems of electromagnetic scattering by a perfectly conducting obstacle. This technique is based on the application of the genetic algorithm (GA). Previous studies have shown that the convergence of the MAS solution depends on many parameters such as the number of auxiliary sources and the auxiliary distance between the real surface of the cylinder and the fictitious surface, and so on. The solution is obtained with a good choice of these parameters. In this article, the genetic algorithm is chosen to facilitate this choice.
\end{abstract}

\section{Introduction}

The Method of Auxiliary Sources (MAS) [1-5] is a numerical method, which has been recently known to be a branch of the generalized multipole techniques (GMTs) [13, 14]. It proposes another way to solve the two-dimensional (2D) and three-dimensional (3D) electromagnetic problem. This method gives very interesting results especially for the electromagnetic scattering problem. Its major advantage comes from the reducing of the complexity of the structure and the minimisation of the computational cost.

However, MAS is not frequently used as the other numerical method, like the Method of Moment (MoM) or the Finite Difference Time Domain (FDTD) [15], due to its limited robustness coming from the ambiguity found in the choice of its parameters.

It is shown in [23] that the convergence of the MAS solution for the scattering problem depends on many parameters. These parameters are the position of the auxiliary surface, the number of auxiliary sources and conditioning number of the system.

For bad position of these parameters, the obtained solution is very bad and characterized by high boundary condition error.

Many studies have been carried out in order to study the best way to determine the suitable values of MAS parameters ensuring a convergent solution like [23].

In [17] the authors studied the convergence of the MAS and showed that, for a limit number of auxiliary sources, it is possible to have a convergent solution for the MAS field with divergent solution for the MAS current. This analysis discussed the effect of the auxiliary sources positions on the boundary condition error and on the condition number of the obtained linear system for the scattering problem.

However, in [18] the authors included a combination using the generalized impedance boundary conditions and Level Set Technique for 3D scattering problems with the MAS in order to find the best positions of the auxiliary sources.

In [22], an application of the GA technique in order to find the location of auxiliary sources giving a minimum value of the boundary condition error, is presented. The principle of this technique is to fix a value of the number of auxiliary sources and then to apply the GA algorithm to obtain the position of these sources giving the low value of error on the boundary of the scatterer. Therefore, the solution does not take into account the matrix conditioning number of the problem.

The convergence of the MAS solution uses the error estimation as the main factor to ensure it.

Besides, in the MAS, the solution of a scattering problem is obtained by representing fields in each domain of the structure under investigation by finite linear combination of the analytical solutions of the sources situated at some distance away from the boundaries of each physical domain. The "auxiliary sources" producing these analytical solutions are chosen to be elementary dipoles, charges, or current configurations located on fictitious auxiliary surface(s), usually conforming to the actual surface(s) of the structure [19]. As it is mentioned the convergence of the MAS does not obeys to any theory or rule.

Many techniques can be applied in the problem of optimization such as the method of genetic algorithm (GA) [21], particle swarm optimization (PSO) method, differential evolution method (DE), etc. In this paper, we propose to optimize the convergence of the MAS with the use of the GA.

This choice is due to the following GA advantages:

- Genetic algorithms use parameter coding, not the parameters themselves.

- Genetic algorithms work on a population of points, instead of a single point.

- Genetic algorithms use only the values of 
the studied function, not its derivative, or some other auxiliary knowledge.

- Genetic algorithms use probabilistic, not deterministic, transition rules.

This paper gives a combination between MAS and GA in order to analyse the convergence of the electromagnetic scattering problem. The solution proposed is tested on an arbitrary cylinder shape. A good agreement is observed with the solution obtained with the standard MAS.

\section{Formulation of the Method of Auxiliary Sources}

Let consider an infinite perfectly conducting cylinder, with an arbitrary surface $\mathrm{S}$. The surface is illuminated by an incident electromagnetic TM plane wave $\left(\mathrm{E}^{\text {inc }}, \mathrm{H}^{\text {inc }}\right)$ (figure 1) expressed as:

$$
\begin{gathered}
E^{i n c}=\mathrm{E}_{0} \exp \left(\mathrm{jk} \mathrm{k}_{0}\left(\mathrm{x} \cos \varphi_{\text {in }}+\mathrm{y} \sin \varphi_{\text {in }}\right)\right) \overrightarrow{\mathrm{z}} \\
H^{i n c}=-\frac{\mathrm{E}_{0}}{\mathrm{H}_{0}}\left(\overrightarrow{\mathrm{x}} \sin \varphi_{\text {inc }}-\right. \\
\left.\overrightarrow{\mathrm{y}} \cos \varphi_{\text {inc }}\right) \exp \left(\mathrm{jk} \mathrm{k}_{0}\left(\mathrm{x} \cos \varphi_{\text {in }}+\mathrm{y} \sin \varphi_{\text {in }}\right)\right)
\end{gathered}
$$

(1)

Where:

- $\quad \mathrm{k}_{0}$ is the vacuum wavenumber

- $\quad \varphi$ in is the incident wave polar angle;

- $\quad x, y$ and $z$ are the unit vector respectively in the $\mathrm{x}, \mathrm{y}$ and direction.

The structure is surrounded by the free space $\left(\varepsilon_{0}, \mu_{0}\right)$, where $\varepsilon_{0}$ is the electric permittivity in free space and $\mu_{0}$ the magnetic permeability in free space.
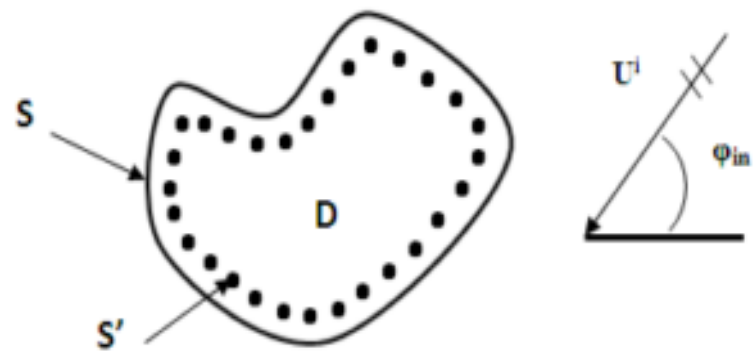

Figure 1: Structure

In the standard MAS, the incident field on the cylinder produces a scattered field. This field is radiated by $\mathrm{N}$ auxiliary sources that are regularly distributed on a fictitious surface inside the PEC cylinder.

Each Auxiliary source radiates an elementary field expressed by:

$$
E_{i}^{\text {scat }}(x, y)=I_{i} H_{0}^{(2)}\left[k_{0} \sqrt{\left(x-x_{i}\right)^{2}+\left(y-y_{i}\right)^{2}}\right] z
$$

Where $\mathrm{I}_{\mathrm{i}}$ is the complex current of the auxiliary sources $\mathrm{i}$ and $H_{0}^{(2)}$ is the zero-order second kind of Hankel function.
The total scattered field is written as:

$$
E_{\text {tot }}^{\text {scat }}(x, y)=\sum_{i=1}^{N} E_{i}^{\text {scat }}
$$

The total electric field is expressed as :

$$
E_{\text {tot }}=E^{\text {inc }}+E^{\text {scat }}
$$

The projection of the equation (4) on the surface $\mathrm{S}$ gives the boundary condition, expressing the continuity of the tangential component of the total electric field, given by:

$$
\vec{n} \times\left(E^{\text {inc }}+E^{\text {scat }}\right)=0
$$

The collocation point method, which is the discretization of the surface $\mathrm{S}$ into $\mathrm{M}$ points, is applied. Therefore, the incident field written on a collocation point $\mathrm{M}\left(\mathrm{x}, \mathrm{ym}_{\mathrm{m}}\right)$ gives the following equation:

$$
\underset{\mathrm{m}=1, \ldots, \mathrm{M}}{E_{m}^{i n c}\left(x_{m}, y_{m}\right)=E_{0} \exp \left\{j k_{0}\left(x_{m} \cos \varphi_{i n}+y_{m} \sin \varphi_{i n}\right)\right\} z}
$$

Now, the combination of equation (2) and equation (6) in equation (5) gives the equation (7):

$$
\begin{aligned}
& \sum_{i=1}^{N} I_{i} H_{0}^{(2)}\left[k_{0} \sqrt{\left(x-x_{i}\right)^{2}+\left(y-y_{i}\right)^{2}}\right]= \\
& -E_{0} \exp \left\{j k_{0}\left(x_{m} \cos \varphi_{i n}+y_{m} \sin \varphi_{i n}\right)\right\}
\end{aligned}
$$

The projection of the equation (7) on $\mathrm{M}$ collocation points gives a linear system when $\mathrm{N}=\mathrm{M}$.

In order to solve the scattering problem, we have to choose the position and the number of the auxiliary sources.

In this paper, we introduce the genetic algorithm to optimize the solution obtained with the MAS.

\section{The Combination MAS/GA}

Genetic Algorithm (GA) is stochastic method, inspirited from the natural biological evolution. This algorithm starts by giving an initial population having $\mathrm{N}$ parents. A crossing between these parents gives different children. An evaluation of the characteristic of these new children is done, in order to the construct a new generation that have the best values of the fitness function of evaluation.

An iterative process is applied from a generation to another until having the best population for a given number of generations.

The combination MAS/GA proposed in this paper, have the objective to determine the number of the auxiliary sources and their positions in order to:

1. minimize the error on the boundary of the cylinder given by the equation (8):

$$
\Delta E_{e r r, S}=\frac{\left|\mathrm{E}^{\mathrm{inc}}+\mathrm{E}^{\text {scat }}\right|}{\max \left\{\left|\mathrm{E}^{\mathrm{inc}}\right|\right\}}
$$

2. Obtain the best conditioning number [23] of the matrix for the linear system.

It is proved in [23] that the error on the boundary of the scattering object decreases when the auxiliary distance 
$\left(d_{\text {aux }}\right)$ value increases. Besides, the conditioning number of the scattering problem matrix increases with the increasing of the $d$ of $d_{\text {aux }}$.

We denote $f_{1}$ : the error on the boundary of the PEC cylinder and $\mathrm{f}_{2}$ : the conditioning number of matrix of the EM scattering problem.

In this paper, we propose to optimise this problem by finding the best combination between all the parameters to ensure the convergence of the solution.

The fitness function (9) consists of two objectives:

$F_{\text {fitness }}=\mathrm{f}\left(\mathrm{f}_{1}, \mathrm{f}_{2}\right)$

The auxiliary sources are placed regularly on a surface $\mathrm{S}^{\prime}$ parallel to $\mathrm{S}$. The distance between $\mathrm{S}$ and $\mathrm{S}$ ' is designed auxiliary distance $d_{a u x}$. So the GA parameters are the number of auxiliary distance $\mathrm{N}$ and $\mathrm{d}_{\text {aux }}$.

To solve a problem with GA, we must first code the parameters, a gene corresponds to an optimization variable $\mathrm{Xi}$, and a set of genes corresponds to a chromosome [11]. An individual corresponds to one or more chromosomes and a population is a set of people.

Every chromosome contains the binary coding of the parameters $\mathrm{N}$ and $\mathrm{d}_{\mathrm{aux}}$.

The application of the GA is started by initialling a population of $\mathrm{N}_{\mathrm{c}}$ chromosomes.

A number of sources and a distance daux define each individual of this population. a calculation of the error on the edges of the cylinder is then carried out by applying the edge conditions.

The same work is done for each generation to find the smallest edge error possible.

The GA proposes operators to pass from one generation to another [2] as given in figure 2:

- Selection: is a very important operator in the process of the genetic algorithm giving the individuals that will be the parents of the next generation. This selection is based on the result of the evaluation of the individual with fitness function. At each generation, the pairs of parent are chosen randomly to give a pair of children.

The selection method used in this paper is to randomly select two individuals and compare their adaptation function (fight) and we accept the most suitable to access the intermediate generation, and we repeat this operation to fill the intermediate generation (number of individuals / 2 components). The individuals who win each time can be copied several times which will promote the durability of their genes. This selection uses a selection probability defined by Holland's roulette wheel.

- Crossovers: this operator generates the children by mixing genetic information from two parents. The obtained chromosomes are constructed by taking the first part of the parent 1 and combined with second part of second part of the parent 2 . The new children are different to all other individuals of the population. The crossover takes place in two stages the selection of the pairs who will become parents and crossing of these new parents who gives two children.

- Mutations: the children obtained from the crossover will be exposed to the operator of mutation. The principal of this operator is to modify the chromosome of the children by changing a bit 0 to 1 or 1 to 0 . The mutation is done with probability in order to look for the best individuals that do not appear with the crossover operation.

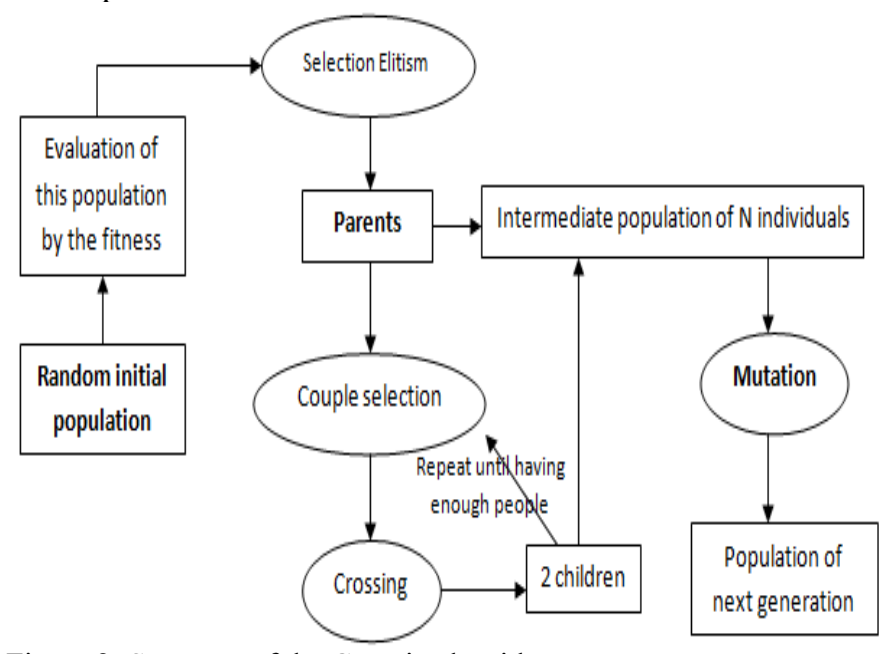

Figure 2: Structure of the Genetic algorithm

\section{The Combination MAS/GA}

In this section, a formulation of the scattering problem with the genetic algorithm is given.

It was demonstrated on the previous sections that boundary conditions error is calculated to ensure the convergence of the solution given by the MAS method.

In order to effectively optimize this problem, a binary coding is chosen. The individuals (chromosomes) are constructed by: $\mathrm{N}_{1}, \mathrm{~N}_{2}, \ldots, \mathrm{N}_{\mathrm{n}} ; \mathrm{d}^{1}{ }_{\text {aux }}, \mathrm{d}^{2}{ }_{\text {aux }}, \ldots, \mathrm{d}_{\text {aux }}^{\mathrm{n}}$

Where:

$\mathrm{N}_{\mathrm{i}}$ is the number of auxiliary sources

$\mathrm{d}_{\text {aux }}^{\mathrm{m}}$ is the auxiliary distance.

The couple $\left(\mathrm{N}_{\mathrm{i}}, \mathrm{d}_{\text {aux }}^{\mathrm{m}}\right)$ is coded by 14 bytes.

The parameters chosen for the GA are:

- $\quad$ Number of individuals $=20$.

- $\quad$ Number of generations $=50$.

- $\quad$ Probability of mutation $=0.05$.

- Probability of crossover $=50 \%$.

In this study, two examples are considered to validate the proposed combination MAS/GA: an infinite PEC cylinder with radius $r=0.6 \lambda$ and a square cylinder with section $=0.7 \lambda$.

The figure (3) shows the average of the values obtained by the fitness function for each generation. 


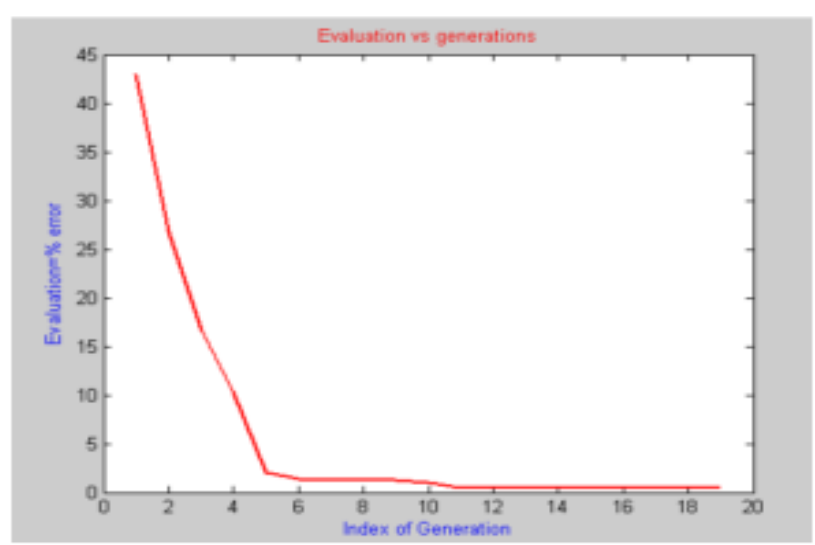

Figure 3: Error evaluation on the boundaries by Genetic algorithm.

\section{Conditioning number vs index of generation}

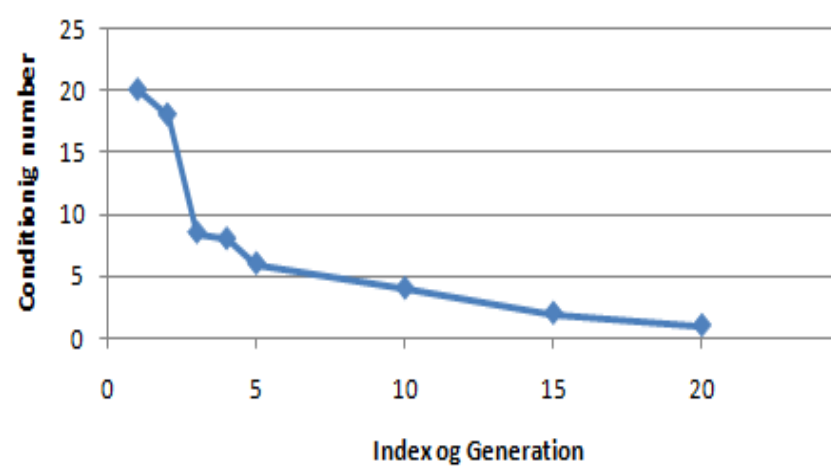

Figure 4: Conditioning Number vs Genetic algorithm

The error calculated for this structure, when considering the values obtained with the combinaison MAS/GA, is given by figure 5 . It shows a good convergence of the solution.

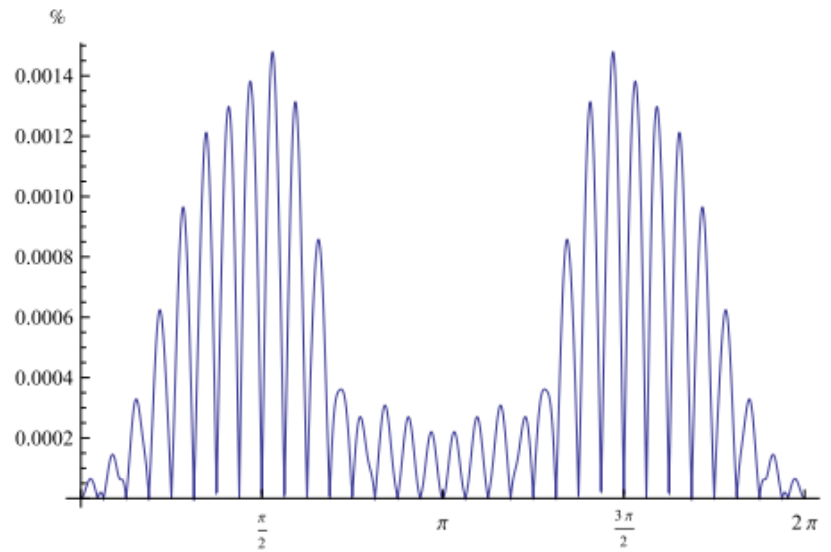

Figure 5: Error in boundary conditions obtained with Genetic Algorithm for the cylinder with radius $r=0.6 \lambda$

The radar cross section (RCS) is defined for $2 \mathrm{D}$ by equation (10) is given in figure 6 for the same cylinder.

$$
S W=\lim _{\rho \rightarrow+\infty}\left[2 \pi \rho \frac{\left|E^{\text {scat }}\right|^{2}}{\left|E^{\text {inc }}\right|^{2}}\right]
$$

The figure (6) gives the radar cross section that simulated for the same structure using the MAS/GA. It shows a good agreement with the solution obtained with standard MAS. Therefore, the solution with MAS/GA is obtained with $\mathrm{N}=16 \mathrm{AS}$ but $25 \mathrm{AS}$ with the MAS.

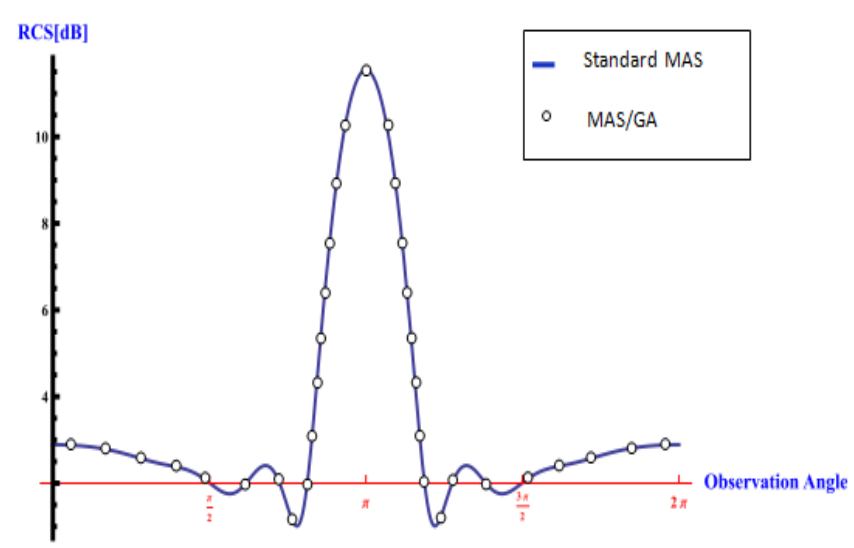

Figure 6: The RCS obtained for the same error in boundary conditions with Genetic Algorithm

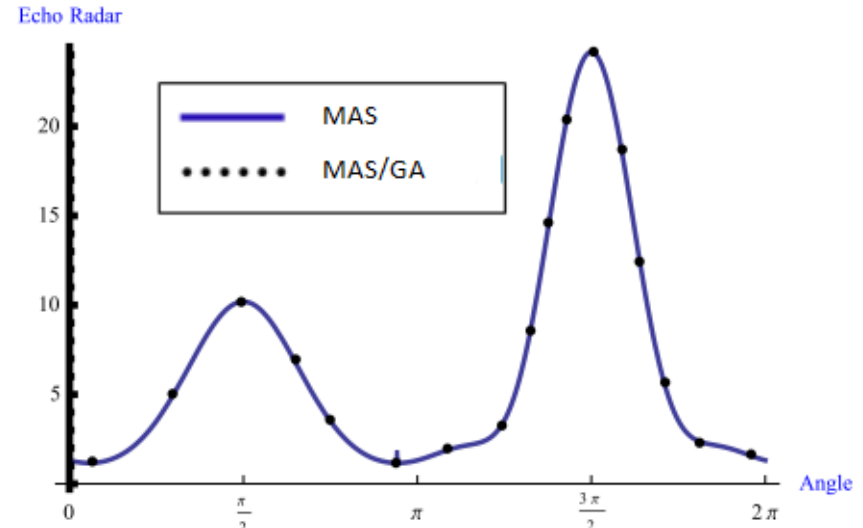

Figure 7: The RCS obtained for the same error in boundary conditions with Genetic Algorithm for square cylinder section $=0.7 \lambda$

The figure 7 represents the radar cross section obtained for a square cylinder illuminated by an incident electromagnetic TM plane wave. A good agreement between the solution obtained by the standard MAS and the MAS/GA is observed.

This result is obtained with 18 AS by the MAS/GA but it is 28 AS with MAS.

The application of the genetic algorithm in the problem of electromagnetic scattering of a monochromatic waveplane by an obstacle gives many generations. A number $\mathrm{N}$ of auxiliary sources and an auxiliary distance characterizes each generation.

The figure 3 shows that error on the boundary of the structure is minimized and the convergence is ensured from the generation 12. However, the conditioning number, given in figure 4, remains important and the accuracy of the solution is not achieved. 
The combination of the solution given in figure 3 and figure 4 allows us to conclude that the solution of electromgnetic scattering problem (circular cylinder radius $r=0.6 \lambda$ ) is convergent from the twentieth generation.

These results make it possible to obtain very small values of the error on the edge conditions of the scatterer.

The evaluation of the MAS / GA method for large obstacles compared to the wavelength also made it possible to give acceptable results. The experiment is made for a $2 \mathrm{D}$ cylinder of radius 9.9 $\lambda$. The convergence of the solution is obtained with the MAS using 185 auxiliary sources. Whereas the same result with the same precision is achieved with MAS / GA using only 150 auxiliary sources.

\section{Conclusion}

In this paper, we present a combination MAS/GA for the optimisation of the $2 \mathrm{D}$ electromagnetic scattering problem by an infinite PEC cylinder. The proposed study includes two objectives for the genetic algorithm that are the error on the boundary of the scatterer and the conditioning number for system's matrix. The obtained solutions show a good agreement with those obtained with the standard MAS.

The advantage of the MAS/GA is reduction of the time lost in groping to find the best values of the MAS parameters that make it possible to find a convergent solution. The MAS/GA gives the position of the auxiliary sources and their number for arbitrary section of scatterers.

\section{References}

[1] Thomas G O, D. I. Kaklamani and H. T. Anstassiu, "Aspects of the Method of Auxiliary Sources (MAS) in computational Electromagnetics," IEEE Antennas and Propagation Magazine, Vol. 44, No. 3, pp. 48-64, June 2002.

[2] R. S. Zaridze, R. Jabava, G. Ahvlediani, and J.Bit Babik, D.Karkashadze, D. P. Economou, and N. K. Uzunoglu, "The method of auxiliary sources and scattered field singularities (Caustics)," J. Electromag. Waves App., vol.12, pp; 1491-1507, 1998

[3] H. T. Anastassiu, V. V. Petrovic, D. I. Kaklamani, and N. K. Uzunoglu, "Analysis of electromagnetic scattering from jet engine inlets using the method of auxiliary sources (MAS)," in Proc. 7th Int. Conf. Advances Communications and Control (COMCON7), Athens, Greece, June Fig. 6. Genetic Algorithm 29July 2, 1999, pp; 217-226

[4] H. T. Anastassiu, D. I. Uzunoglu, "The method of auxiliary sources (MAS) applied to radar cross section (RCS) evaluation of jet engine inlets," in Proc. 2000 USNC/URSI Meeting Dig., Salt Lake City, UT, p. 391.

[5] H. T. Anastassiu, D. I. Kaklamani, D. P. Economou, and O. Breinbjerg, "Electromagnetic scattering analysis of coated conductors with edges using the method of auxiliary sources (MAS) in conjunction with the standad impedance boundary condition (SIBC)," IEEE
Tran. Antennas Propagat., vol. 50, pp. 59-66, Tan.2002.

[6] R Y. Leviatan, D.-H. Shan, and A. Adams, "Numerical study of the current distribution on a post in a rectangular waveguide," IEEE Trans. Microwave Theory Tech., vol. MMT-32, pp. 1411-1415, Oct. 1984.

[7] Y. Leviatan, and A. Boag, "Analysis of electromagnetic scattering from dielectric cylinders using a multifilament current model, "IEEE Trans. Antennas Propagat., vol. 35, pp. 1119-1127, 1987.

[8] Y. Leviatan, "Analytic continuation considerations when using generalized formulations for scattering problems," IEEE Trans. Antennas Propagat., vol. 38, pp. 1259-1263, Aug. 1990.

[9] Doicu,A.,Y u. Eremin, and T. Wriedt, "Acoustic and Electromagnetic Scattering Analysis Using Discrete Sources", Academic Press, New York,2000.

[10] Fairweather, G., A. Karageorghis, and P. A. Martin, "The method of fundamental solutions for scattering and radiation problems," Engineering Analysis with Boundary Elements, Vol. 27,759-769, 2003.

[11] Golberg, M. A. and C. S. Chen, "The method of fundamental solutions for potential, Helmholtz and diffusion problems," Boundary Integral Methods and Mathematical Aspects, M. A. Golberg (ed.),103-176, WIT Press/Computational Mechanics Publications, Boston, 1999.

[12] G. Tayeb, R. Petit, and M. Cadilhac. "The synthesis method applied to the problem of diffraction by gratings: the method of fictitious sources," Internat. Conf. Appl. Theory Periodic Structures SPIE, volume 1545, pages 95-105.

[13] Bogdanov, F. G., D. D. Karkashadze, and R. S. Zaridze, "The method of auxiliary sources in electromagnetic scattering problems," Generalized Multipole Techniques for Electromagnetic and Light Scattering, T. Wriedt (ed.), Elsevier Science B. V., 1999.

[14] Kaklamani, D. I. and H. T. Anastassiu, "Aspects of the method of auxiliary sources (MAS) in computational electromagnetics," IEEE Antennas and Propagation Magazine, Vol. 44, No. 3, 48-64, 2002.

[15] Akyurtlu, A. and D. H.Werner, "Novel dispersive FDTD formulation for modeling transient propagation in chiral metamaterials," IEEE Transactions on Antennas and Propagation, Vol. 52, No. 9, 2267-2276, September 2004.

[16] Demir, V., A. Z. Elsherbeni, and E. Arvas, "FDTD formulation for dispersive chiral media using the $\mathrm{Z}$ transform method," IEEE Transactions on Antennas and Propagation, Vol. 53, No. 10, 3374-3384, October 2005.

[17]H. T. Anastassiu, "Error Estimation of the Method of Auxiliary sources (MAS) for Scattering from an Impedance Circular Cylinder" Progress In Electromagnetics Research, PIER 52, 109128, 2005

[18] Afif Bouzidi and Taoufik Aguili, "Optimization of the Method of Auxiliary Sources for 3D Scattering Problems by Using Generalized Impedance Boundary 
Conditions and Level Set Technique", Progress In Electromagnetics Research B, Vol. 61, 121133, 2014.

[19] Fridon Shubitidze, Kevin O’Neill, Shah A. Haider, Keli Sun, and Keith D. Paulsen, "Application of the Method of Auxiliary Sources to the Wide-Band Electromagnetic Induction Problem," IEEE Transactions on Antennas and Propagation, Vol.40, No. 4, 3374-3384, April 2002

[20]Hristos T.Anastassiu, Dimitrios G. Lymperopoulos, and Dimitra I. Kaklamani, "Accuracy Analysis and Optimization of the Method of Auxiliary Sources (MAS) for Scattering by a Circular Cylinder", IEEE Transactions on antennas and Propagation, VOL. 52, NO. 6, JUNE 2004

[21] J.H. Holland, "Adaptation in Natural and Artificial Systems", 0.0012 Cambridge, MA: The M.I.T. Press, (1975).

[22] I. I. Heretakis, P. J. Papakanellos, C. N. Capsalis, "Analysis of electromagnetic scattering by infinite conducting cylinders of arbitrary smooth cross section using a genetically optimised technique (GA/MAS)," Journal of electromagnetic waves and applications, vol. 16, pp. 1555-1572, 2002.

[23] Sami Hidouri, Messaoud Eljamai, Taoufik Aguili, "Study on the parameters for the convergence of the Method of Auxiliary Sources" IEEE - 15th International Wireless Communications and Mobile Computing Conference (IWCMC). 24-28 June 2019, Tangier, Morocco. 